\title{
Von Georg II. zu George Washington: Überlegungen zur Visualisierung von Legitimität im Übergang von Monarchie zu Demokratie ${ }^{1}$
}

\author{
Volker Depkat
}

\section{Einleitung}

Die Kurfürsten von Hannover waren als Könige von England im Zeitalter der Personalunion auch Herren über ein weitverzweigtes Kolonialreich, dessen Schwerpunkt bis 1776 in Nordamerika lag. ${ }^{2}$ Für die Bewohner der britischen Kolonien in Nordamerika waren Georg I., Georg II. und Georg III. die Oberhäupter eines imperialen Herrschaftsverbandes, in den sie hineinwuchsen, der ihr Alltagsleben strukturierte und der auch ihre koloniale Identität bestimmte. Dabei hatten die nordamerikanischen Kolonisten durchaus ihr Scherflein dazu beigetragen, dass England in einer Reihe von Kolonialkriegen gegen Frankreich bis 1763 zum Herrn über den nordamerikanischen Kontinent aufsteigen konnte, und sie sonnten sich als überzeugte Englishmen nur allzu gerne im imperialen Glanz des britischen Weltreiches. ${ }^{3}$

1 Ich bedanke mich bei Tamara Heger und Katharina Matuschek für die Mithilfe bei diesem Aufsatz.

2 Zum britischen Weltreich hier nur: Peter Wende, Das Britische Empire. Geschichte eines Weltreichs, München 2008; Hermann Wellenreuther, Der Aufstieg des ersten Britischen Weltreiches. England und seine nordamerikanischen Kolonien 1660-1763, Düsseldorf 1987; Ders., Niedergang und Aufstieg. Geschichte Nordamerikas vom Beginn der Besiedlung bis zum Ausgang des 17. Jahrhunderts, Münster 2000; Ders., Ausbildung und Neubildung. Die Geschichte Nordamerikas vom Ausgang des 17. Jahrhunderts bis zum Ausbruch der Amerikanischen Revolution 1775, Hamburg 2001; Alan Taylor, American Colonies. The Settling of North America, New York 2001; Jack P. Green / J. R. Pole, Colonial British North America. Essays in the New History of the Early Modern Era, Baltimore 1984.

3 Zur Rolle der Kolonialkriege und ihre Bedeutung für das Selbstverständnis der Kolonisten: Taylor, 2001 (wie Anm. 2), S. 420-443; Wellenreuther, 1987 (wie Anm. 2), S. 265-298; Fred Anderson, Crucible of War. The Seven Years' War and the Fate of Empire in British North America, 1754-1766, New York 2000. 
Diese imperiale Identität war auch für diejenigen attraktiv, die aus anderen, europäischen Ländern in die nordamerikanischen Kolonien einwanderten. Nehmen wir zum Beispiel Heinrich Melchior Mühlenberg, der, 1711 in Einbeck geboren, im Jahr 1741 von Halle aus als lutherischer Pastor nach Pennsylvania entsandt wurde, um dort die lutherische Kirche aufzubauen, und dies auch sehr erfolgreich tat. Er war Zeit seines Lebens stolz darauf, ein Untertan des Kurfürsten von Hannover und Königs von England zu sein. Dafür musste er im 18. Jahrhundert noch nicht einmal die Dynastie wechseln. ${ }^{4}$

Allerdings war das 18. Jahrhundert auch die Zeit, in der große Teile Nordamerikas für die britische Monarchie verloren gingen, weil sich dreizehn Kolonien am 4. Juli 1776 für unabhängig erklärten und die Vereinigten Staaten von Amerika gründeten, die dann im weiteren Verlauf ihrer Geschichte Großbritannien aus Nordamerika verdrängten und selbst zur Weltmacht wurden. ${ }^{5}$

Die sich während der Regentschaft Georgs III. ereignende Amerikanische Revolution steht am Anfang der politischen Moderne. ${ }^{6}$ Sie realisierte ein rein säkular begründetes Gemeinwesen, das das Volk zum Souverän und Autor einer geschriebenen Verfassung hatte, die alle legitime politische Macht aus der Gesellschaft selbst hervorgehen ließ und die Macht der Herrschenden vom Vertrauen und der Zustimmung des Volkes abhängig machte. Die Begründung und Ausgestaltung dieser politischen Ordnung ist ein sehr aufregender und höchst komplizierter Prozess, der auf verschiedenen Ebenen ablief - und auf jeder gleichermaßen spannend ist.

$\mathrm{Da}$ ist zunächst die verfassungsrechtliche Dimension, bei der es darum ging, die in der Unabhängigkeitserklärung verkündeten politisch-sozialen Prinzipien in eine institutionelle Ordnung zu überführen, die das Grundrecht auf life, liberty and the pursuit of happiness wie eine sich selbst-bewegende Maschine auf alle Ewigkeit garantieren würde. ${ }^{7}$ Auch hatte die Ausgestaltung der amerikanischen Demokratie

4 Zu Mühlenberg: Thomas J. Müller, Kirche zwischen zwei Welten. Die Obrigkeitsproblematik bei Heinrich Melchior Mühlenberg und die Kirchengründung der deutschen Lutheraner in Pennsylvania, Stuttgart 1994; Jürgen Gröschl / Thomas Müller-Bahlke / Claus Veltmann (Hg.), Freiheit, Fortschritt und Verheißung. Blickwechsel zwischen Europa und Nordamerika, Halle an der Saale 2011.

5 Zur Amerikanischen Revolution: Francis D. Cogliano, Revolutionary America, 1765-1815. A Political History, New York 22009; Robert Middlekauff, The Glorious Cause. The American Revolution, 1763-1789, New York 1982; Gordon S. Wood, The Radicalism of the American Revolution, New York 1992; Hermann Wellenreuther, Von Chaos und Krieg zu Ordnung und Frieden. Der Amerikanischen Revolution Erster Teil, 1775-1783, Berlin 2006.

6 Zum Zusammenhang von Aufklärung, Revolution und Moderne: Jonathan I. Israel, A Revolution of the Mind. Radical Enlightenment and the Intellectual Origins of Modern Democracy, Princeton, N. J. 2010; Ders., Democratic Enlightenment. Philosophy, Revolution, and Human Rights 1750-1790, New York 2011; Frank Kelleter, Amerikanische Aufklärung. Sprachen der Rationalität im Zeitalter der Revolution, Paderborn 2002.

7 Zur Verfassungsgeschichte der Amerikanischen Revolution: Richard R. Beeman / Stephen Botein / Edward C. Carter (Hg.), Beyond Confederation: Origins of the Constitution and American National Identity, 
eine soziale Dimension, galt es doch auszuhandeln, welche sozialen Gruppen in einer Ordnung, die auf einem radikalen Gleichheitsgrundsatz aufgebaut war (we hold these truths to be self-evident that all men are created equal), denn nun eigentlich das Sagen haben sollten. ${ }^{8}$ Dann ist da noch die identitätspolitische Ebene, denn der Prozess der Loslösung von England und die Begründung eines neuen, als ideal und exzeptionell antizipierten Nationalstaats beinhaltete auch einen tiefen Wandel in den Köpfen der Bewohner der britischen Kolonien in Nordamerika, die bis weit in die 1770er Jahre hinein von sich als ,Engländer ${ }^{`}$ zu denken geneigt waren und sich nun plötzlich als Amerikaner definieren mussten. What do we mean by the revolution?, fragte John Adams rückblickend in einem Brief an Thomas Jefferson im Jahr 1815. The war? That was no part of the revolution; it was only an effect and consequence of it. The revolution was in the minds of the people, and this was effected from 1760 to 1775, in the course of fifteen years, before a drop of blood was shed at Lexington. ${ }^{9}$ Schließlich ging es bei Gründung und Ausgestaltung der amerikanischen Demokratie auch um die Erfindung einer politischen Kultur, die die revolutionär begründete Verfassungsordnung tragen und stabilisieren würde. Dieser Aspekt steht im Zentrum des vorliegenden Beitrags.

\section{Die Politik des Visuellen - Visuelle Politik}

Unter ,politischer Kultur' soll hier die Gesamtheit aller Symbole, Wertideen und Praktiken verstanden werden, die die Legitimität einer politischen Ordnung sowie die Autorität ihrer Amtsträger begründen und dem politischen Handeln in dieser Ordnung Orientierung geben. ${ }^{10}$ Ein zentrales Feld ,politischer Kultur ${ }^{6}$ ist in diesem Zusammenhang der gesamte Bereich des Visuellen, denn Herrschaftsordnungen können nur dann Legitimität und Gültigkeit für sich beanspruchen, wenn sie sich der normativen, wertideellen Grundlagen bewusst sind, auf denen ihre Autorität und ihre regulativen Funktionen beruhen. Politische Systeme sind deshalb nur solange stabil,

Chapel Hill, N.C. 1987; Gordon S. Wood, The Creation of the American Republic, 1776-1787, Chapel Hill, N. C. 1969; Michael G. Kammen, A Machine that would go of itself. The Constitution in American Culture, New York 1986.

8 Zum egalitären Radikalismus der Amerikanischen Revolution und dem konservativen Impetus der Eindämmung der egalitären Energien insbesondere: Wood, 1992 (wie Anm. 5); Ders., 1969 (wie Anm. 7), S. 471-564.

9 John Adams and Thomas Jefferson, 24. August 1815, in: The Works of John Adams, Second President of the United States. With a Life of the Author, Notes and Illustrations, by his Grandson Charles Francis Adams, Bd. 10, Boston 1856, S. 172.

10 Karl Rohe, Politische Kultur und ihre Analyse. Probleme und Perspektiven der politischen Kulturforschung, in: Historische Zeitschrift 250 (1990), S. 321-346; Barbara Stollberg-Rilinger (Hg.), Was heißt Kulturgeschichte des Politischen?, Berlin 2005; Wolfgang Hardtwig (Hg.), Ordnungen in der Krise. Zur politischen Kulturgeschichte Deutschlands 1900-1933, München 2007. 
wie die Normen und Wertideen, auf denen ihr institutionelles Gefüge beruht, erkennbar sind und sichtbar gemacht werden können. Kurz, politische Systeme müssen die normativen und wertideellen Grundlagen ihrer Ordnung visualisieren können. Das gilt auch - und vielleicht sogar insbesondere - für demokratische Ordnungen, die im Prinzip der Volkssouveränität gründen. ${ }^{11}$

In diesem Zusammenhang nun erhalten Bilder von Politikern - zumal die von Regierungschefs und Staatsoberhäuptern - eine zentrale politische Bedeutung, denn, diese These wird hier jedenfalls vertreten, in diesen Bildern werden die Normen und Wertideen einer politischen Ordnung in besonderem Maße sichtbar gemacht. Dies geschieht entweder in affirmativer Absicht oder aber mit der Absicht der Infragestellung, der Kritik oder der Negation eines normativen Geltungsanspruches. Insofern ordnet sich der vorliegende Beitrag in das Feld einer kulturgeschichtlich erweiterten Politikgeschichte ein, die das Visuelle als integralen Bestandteil von Politik begreift, „Bilder der Macht" analysiert und die „Macht der Bilder“ anerkennt. ${ }^{12}$ Dabei weiß er sich methodisch den Visual Culture Studies verpflichtet, deren wesentliche Prämissen hier in vier Punkten kurz vorgestellt seien. ${ }^{13}$

Bilder sind erstens nicht nur bloße Illustrationen von Texten, sondern eine ganz eigene Form der Ordnung, Interpretation und Aneignung von Welt, die gleichberechtigt neben Texten steht und ihre ganz eigene Dynamik hat. Bilder lassen sich mithin als Akte kultureller Sinnstiftung begreifen, die jene orientierungsgebenden Perspektiven auf die Welt organisieren, ohne die intentionales Handeln in ihr nicht möglich ist. Bilder sind deshalb nicht nur ein gleichsam passiver Spiegel der äußeren Wirklichkeit, sondern sie bringen die Wirklichkeit, die sie darstellen, immer auch ein Stück weit mit hervor. Als solche Repräsentationen von Wirklichkeit sind Bilder zweitens auch als visuelle Argumente zu begreifen, die integrale Bestandteile von denjenigen Prozessen sozialer Kommunikation sind, durch die sich einzelne Gruppen und ganze Gesellschaften über sich selbst und ihren Ort in der Welt verständigen. ${ }^{14}$ Drittens lassen Bilder sich immer auch als Visualisierung von abstrakten Wertideen

11 Wolfram Pyta, Visualizing Democratic Legitimacy and Authority. The Case of the Weimar Republic, in: Volker Depkat / Meike Zwingenberger (Hg.), Visual Cultures - Transatlantic Perspectives, Heidelberg 2012, S. 69-83, hier S. 69f.

12 Stefan Germer / Michael F. Zimmermann (Hg.), Bilder der Macht, Macht der Bilder. Zeitgeschichte in Darstellungen des 19. Jahrhunderts, München 1997.

13 Nicholas Mirzoeff, An Introduction to Visual Culture, New York 22010; Ders., The Visual Culture Reader, London ${ }^{3}$ 2013; Gunther Kress / Theo van Leeuwen, Reading Images. The Grammar of Visual Design, London 22010; Depkat / Zwingenberger, 2012 (wie Anm. 11).

14 Zum Konzept der sozialen Kommunikation: Volker Depkat, Kommunikationsgeschichte zwischen Mediengeschichte und der Geschichte sozialer Kommunikation. Versuch einer konzeptionellen Klärung, in: Karl-Heinz Spieß (Hg.), Medien der Kommunikation im Mittelalter, Stuttgart 2003, S. 9-48; Demnächst auch: Ders., Wege in die Kommunikationsgeschichte des Zeitalters der Personalunion, in: Steffen Hölscher / Sune Schlitte (Hg.), Kommunikation im Zeitalter der Personalunion (1714-1837). Prozesse, Praktiken, Akteure, Göttingen (im Druck). 
und Normen einer Kultur lesen. Sie machen etwas Unsichtbares wie beispielsweise ,die Nation“ oder ,den Staat‘ oder eben auch ,Legitimität‘ oder ,Demokratie“ sichtbar. Dabei folgen sie viertens einer kulturell konditionierten und deshalb auch kulturell spezifischen „visuellen Grammatik“, also einem Regelsystem, das bestimmt, wie Bilder aufgebaut sind. ${ }^{15}$ In diesem Zusammenhang kann man danach fragen, welche Bedeutung den Farben zukommt, welchen Informationsgehalt die verschiedenen Zonen eines Bildes haben, wie Rahmen gesetzt werden, aus welchen Elementen Bilder bestehen und wie diese zueinander in ein Verhältnis gesetzt werden. Die kulturell bestimmte Grammatik des Visuellen freizulegen, heißt Bilder ,lesen zu lernen`.

Auf dieser Grundlage steht der vorliegende Beitrag, der es sich zum Ziel gesetzt hat, eine Auswahl von Porträts der hannoversch-britischen Könige mit denen von George Washington zu vergleichen, der als erster postrevolutionärer Staats- und Regierungschef am Anfang des von Alexis de Tocqueville diagnostizierten „Zeitalters der Demokratie“ steht. ${ }^{16}$ Wie also haben europäische Zeitgenossen Könige gemalt, um königliche Herrschaft zu legitimieren? Wie haben dann die Amerikaner ihre Präsidenten gemalt, um Macht und Autorität eines demokratisch gewählten Staatsoberhaupts und Regierungschefs zu legitimieren? Wie hat - damit einhergehend - die frühe amerikanische Demokratie ihren Anspruch auf Legitimität und Autorität in einem historischen Kontext visualisiert, in dem die Visualität politischer Macht entscheidend von monarchisch-aristokratischen Traditionen geprägt war, in die die Kolonisten Britisch-Nordamerikas selbst hineingewachsen waren?

\section{Die Visualisierung monarchischer Legitimität in der Personalunion}

Wenn die Bewohner der dreizehn britischen Kolonien sich am Vorabend ihrer Revolution vorstellten, wie ,politische Macht' denn aussehe und woran man sie erkenne, dann hatten viele von ihnen ganz bestimmt die Porträts der britischen Könige vor Augen, deren Konterfeis in Form von Gemälden und Stichen auch in den Kolonien überall angebracht waren. Von besonderer Bedeutung waren in diesem Zusammenhang zwei Formen des Monarchenporträts, nämlich zum einen Krönungsbilder, die als offizielle Staatsporträts die Macht des Königs in besonders verdichteter Form repräsentierten, und zum anderen Darstellungen des Monarchen als Feldherren.

15 Zur visuellen Grammatik speziell: Kress / van Leeuwen, 2010 (wie Anm.13).

16 Isaac Kramnick (Hg.), Alexis de Toqueville. Democracy in America and Two Essays on America, übers. von Gerald E. Bevan, London 2003. 
Eines dieser Krönungsbilder befindet sich seit 1837 an der sogenannten Königswand im Aula-Gebäude der Göttinger Universität (Abb. 1). Es ist das lebensgroße Porträt von König Georg II., dem Stifter der Universität, das 1741 vom hannoverischen Hofmaler Gottfried Boy gemalt wurde und zunächst in Hannover hing, bevor es 1746 auf Bitten der Universität nach Göttingen überführt wurde. Dort hing es bis 1837 in verschiedenen Räumen und fand dann mit Eröffnung der neuen Aula seinen endgültigen Platz. ${ }^{17}$

Dieses Bild König Georgs II. ist ein konventionelles Staatsporträt, das die Macht und Legitimität des britischen Königs und Kurfürsten von Hannover vor allem durch den Körper des Königs und die materiellen Objekte im Raum visualisiert. ${ }^{18}$ Werfen wir zunächst einen Blick auf den Körper des Königs, dem seit dem Mittelalter eine zentrale Bedeutung für die Visualisierung königlicher Macht zukam. ${ }^{19}$ Der gemalte Georg II. ist von offensichtlich guter Statur. Er steht aufrecht in voller Beherrschung des eigenen Körpers. Sein Gesicht ist ebenmäßig, fast engelsgleich. Im Körper des Königs sind Selbstbeherrschung, Kraft und auch männliche Schönheit repräsentiert. Dieser Körper ist bekleidet, und zwar mit erkennbar kostbarem Geschmeide in den Farben der Macht. Georg II. trägt einen purpurnen, mit Goldbrokat und Hermelin besetzten Gehrock und einen purpurnen Umhang, dessen Innenseite ganz aus Hermelin ist, dazu silbrig glänzende Schnallenschuhe sowie weiße Strümpfe und eine Allongeperücke. Insgesamt ist der Glanz der Kleidung verschwenderisch. Sie verbindet sich harmonisch mit der Statur des königlichen Körpers, und beides zusammen lässt den Monarchen zu einer nicht mehr ganz irdischen Erscheinung werden.

Genauso wichtig - ja fast noch wichtiger - für die Repräsentation königlicher Macht, sind die im Raum verstreuten, aber doch mit dem Körper des Königs verbundenen materiellen Objekte als Insignien der Macht. Wir sehen die Krone auf dem Tisch links hinter dem König. Er hält ein Zepter in der Hand, und sein Umhang liegt zum Teil auf dem Thronsessel im rechten Bildhintergrund. Alle diese Objekte verweisen auf ,Blut' und ,Gottesgnadentum' als die beiden Quellen monarchischer Legitimität. Hinzu kommt der Habitus des Abgehobenen. Georg II. steht auf einem Podest unter einem Baldachin in einem geschlossenen Raum. Es kommt zu keinem direkten Blickkontakt mit dem Betrachter. Der Monarch schaut aus dem Bild heraus über den Betrachter hinweg auf etwas, das weit in der Ferne liegt. Der König ist buchstäblich entrückt. Er steht in keinem Kontakt mit der Welt da draußen. Er ist nicht

17 Marianne Bergmann / Christian Freigang, Das Aula-Gebäude der Göttinger Universität. Athen im Königreich Hannover, München 2006, S. 43.

18 Zu Georg II. hier nur: Mijndert Bertram, Georg II. König und Kurfürst, Göttingen ${ }^{2} 2004$.

19 Ernst H. Kantorowicz, Die Zwei Körper des Königs. Eine Studie zur politischen Theologie des Mittelalters, übers. von Walter Theimer, Stuttgart 1992; Florentine Mütherich (Hg.), Percy Ernst Schramm, Die deutschen Kaiser und Könige in Bildern ihrer Zeit 751-1190, München 1983. 


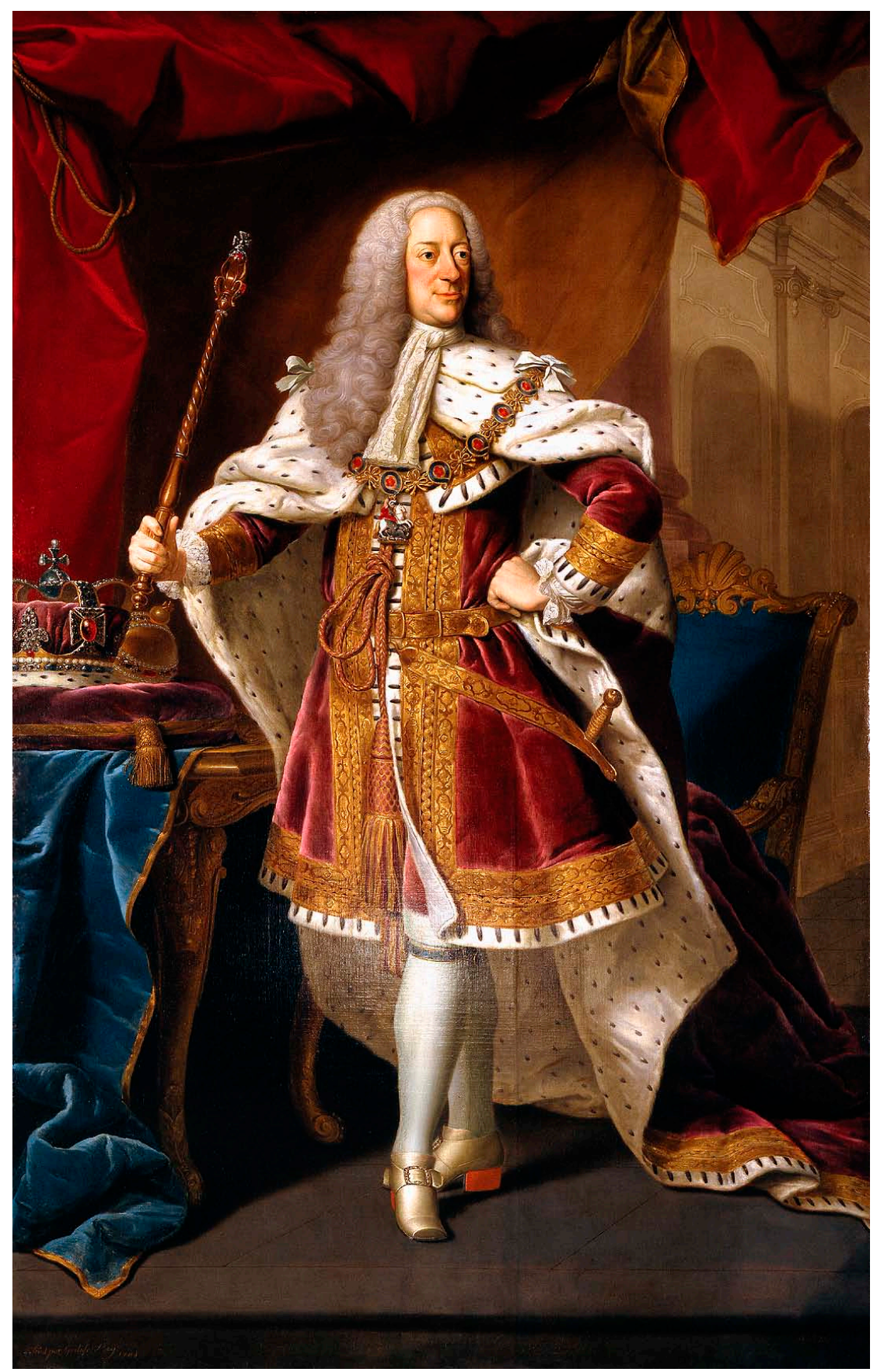

Abb. 1: Gottfried Boy, König Georg II., 1741.

Aula-Gebäude der Göttinger Universität. 
Teil der Gesellschaft, die er regiert, sondern schwebt irgendwo zwischen der irdischen und der göttlichen Sphäre.

So konventionell diese Darstellung des abgehobenen Königs als Quelle aller politischen Macht auch sein mag, sie ist insofern bemerkenswert, als der König in Großbritanniens gemischter Verfassung um die Mitte des 18. Jahrhunderts deutlich an Bedeutung verlor. Zwar war die Macht der Könige weiterhin substantiell, doch gewann die Staatsbürokratie und vor allem der Premierminister in dieser Zeit massiv an Einfluss. Kein Geringerer als Georg II. höchstselbst stellte 1744 resigniert fest, dass die Minister die eigentlichen Könige des Landes seien. ${ }^{20}$ Insofern lässt sich diese Darstellung scheinbar uneingeschränkter königlicher Machtvollkommenheit auch als visuelle Behauptung eines Herrschaftsanspruches lesen, der durch den Aufstieg der modernen Staatsverwaltung im Großbritannien des 18. Jahrhunderts tatsächlich immer problematischer wurde.

Deutet das bislang erörterte Krönungsbild Georgs II. auf ,Blut' und ,Gottesgnadentum' als Quellen königlicher Macht, so verweisen Bilder, die den König als Feldherren darstellen, auf militärisches Charisma als eine weitere wichtige Säule des monarchischen Herrschaftsanspruchs.

Von besonderer Bedeutung sind in diesem Falle Reiterbilder, wie das von Joseph Highmore, ein damals gefragter Porträtist, Maler historischer Szenen und Freund von William Hogarth (Abb. 2). Das Mitte der 1740er Jahre entstandene Reiterbild war offenbar eine Vorstudie für ein geplantes, aber nie realisiertes lebensgroßes Reiterporträt von Georg II. ${ }^{21}$ Diese Darstellung Georgs II. stand in der ikonographischen Tradition des Reiterbildes von Karl I., das Anthony van Dyck 1633 angefertigt hatte, welches wiederum sein Vorbild in Tizians Reiterporträt von Karl V. aus dem Jahr 1548 fand. Das zentrale Thema ist hier das vom König als Feldherren, dessen Autorität und Charisma auf militärischen Erfolgen beruht. Das Bild zeigt Georg II. in voller Ritterrüstung wie er hoch zu Ross unter einem antiken Triumphbogen hervorreitet. In der rechten Hand hält er einen Feldherrenstab als Zeichen seiner Autorität. Hinter dem Pferd des Königs ist ein Page zu sehen, der den gefiederten Helm des Königs trägt, was die Referenzen ans mittelalterliche Rittertum noch verstärkt. Im rechten Bildhintergrund kann man unten Kavalleriesoldaten erkennen, die auf ihren von einer Anhöhe herabreitenden Oberkommandierenden warten. Insgesamt handelt es sich hier um ein antikisierend-mediävistisches Phantasiebild von Georg II. als Feldherrn, das auf die feudalen Grundlagen königlicher Legitimität verweist.

Interessant ist nun, dass Georg II. der letzte britische König war, der tatsächlich noch selbst als Militärmonarch agiert hat: In der Schlacht von Dettingen komman-

20 John Cannon / Ralph Griffith, The Oxford Illustrated History of the British Monarchy, New York 1988, S. 473.

21 Martin Postle, Joseph Highmore. Equestrian Portrait of King George II. https://www.tate.org.uk/art/artworks/highmore-equestrian-portrait-of-king-george-ii-t04944/text-summary (letzte Einsicht: 30.03.2014). 


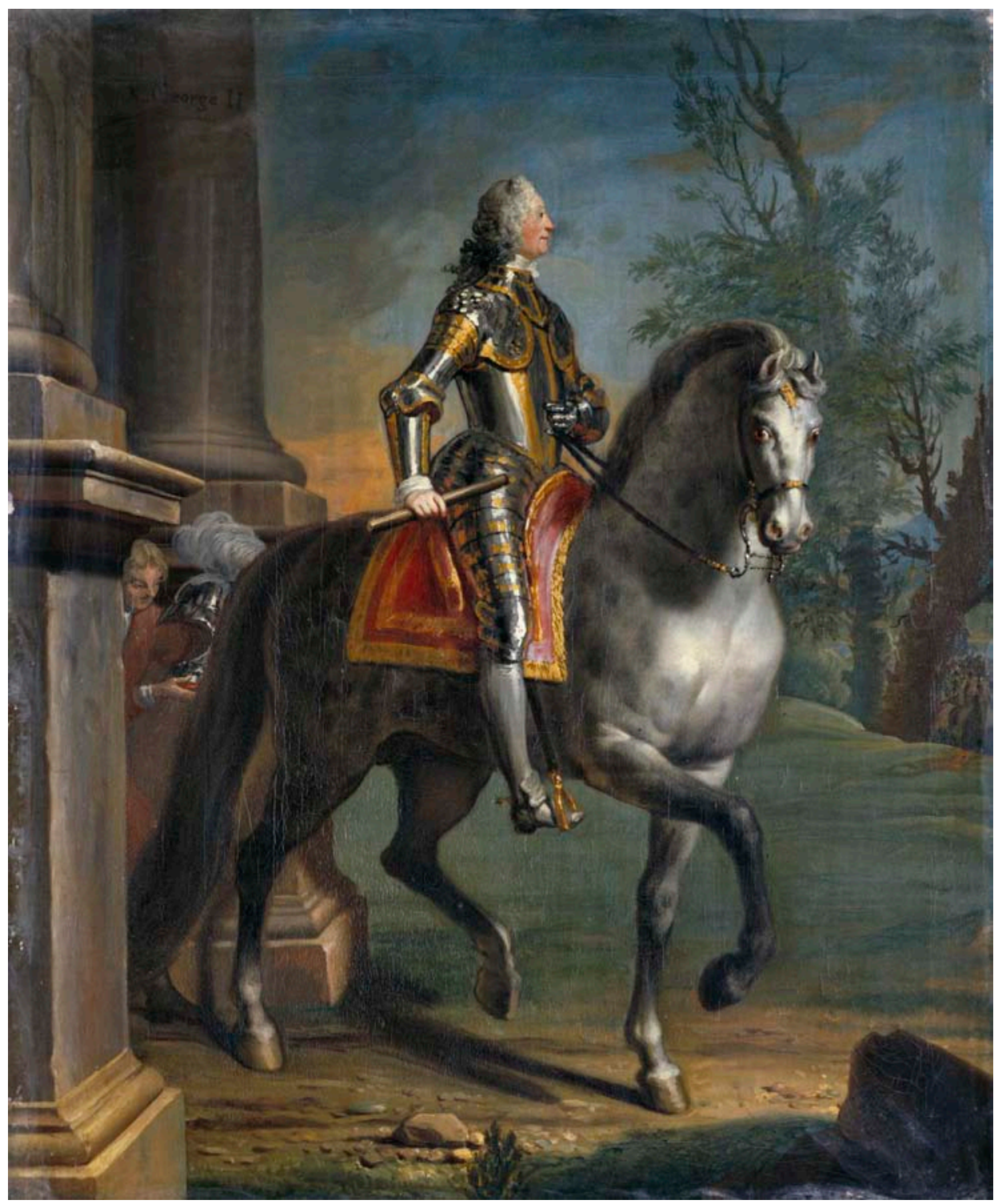

Abb. 2: Joseph Highmore, Reiterporträt von König Georg II., ca. 1743-1745. Tate Gallery, T04944.

dierte er im Jahr 1743 höchstpersönlich die hannoverischen und britischen Truppen zum Sieg über die Franzosen, was ihm bei seinen fremdelnden britischen Untertanen einen großen Popularitätsschub bescherte. Sein Nachfolger, Georg III., spielte die Rolle als Oberkommandierender der Truppen wegen der damit verbundenen Risi- 
ken nicht mehr persönlich, sondern nur noch virtuell. ${ }^{22}$ In der Folge setzte sich die Haltung durch, das Leben des Thronfolgers nicht durch militärische Einsätze zu gefährden und stattdessen den Zweitgeborenen mit der Aufgabe als commander-in-chief der britischen Truppen zu betrauen. In der Ikonographie monarchischer Macht blieb der König als Feldherr - oft in Form eines Reiterstandbildes - jedoch weiterhin sehr präsent.

\section{Der Anti-Monarchismus der Amerikanischen Revolution}

Die Amerikanische Revolution begann nach dem Ende des Siebenjährigen Krieges im Jahr 1763 als Steuerstreit zwischen dem Mutterland und seinen Kolonien, der sich bis Mitte der 1770er Jahre im Rahmen der britischen Verfassung entfaltete. ${ }^{23}$ In diesem Streit ging es um die Frage, wer das Recht hatte, in den Kolonien Britisch-Nordamerikas Steuern zu erheben, das britische Parlament in London oder die in den einzelnen Kolonien lokal gewählten Parlamente. Ausgelöst wurde er durch eine Reihe von Steuergesetzen, die das britische Parlament im Laufe der 1760er Jahre beschloss und sich damit kühl über die bereits im Vorfeld geäußerte massive Kritik der Kolonisten hinwegsetzte. Die Bewohner der nordamerikanischen Kolonien waren nicht bereit, die von London beschlossenen Steuergesetze zu akzeptieren, weil sie der Meinung waren, dass nur die von ihnen selbst gewählten Kolonialparlamente dies dürften. Dabei beriefen sich die nordamerikanischen Kolonisten in ihrem Protest gegen die britischen Steuergesetze auf ihre Rights of Englishmen, auf diejenigen Rechte, die sie ihrer Überzeugung nach als Engländer hatten. Als Engländer, so meinten sie, dürften sie nicht von einem Parlament besteuert werden, in dem sie nicht selbst auch repräsentiert wären. Anfangs protestierten die Bewohner der britischen Kolonien in Nordamerika also als ,Engländer' gegen die britische Regierung in London, die aus ihrer Sicht die angestammte britische Verfassungsordnung verletzte.

Dabei blieb es jedoch nicht. In dem Maße, in dem der Streit eskalierte, weil London sich unversöhnlich zeigte und mit harter Hand gegen die aufsässigen Kolonisten vorging, schwenkten die Kolonisten auf eine neue Linie ein. Die ,revolutionäre Wende' der Amerikanischen Revolution fällt in die Jahre von 1774/76. Das Revolutionäre an dieser Wende ist, dass die amerikanischen Kolonisten in diesen Jahren aus

22 Clarissa Campbell Orr, The Feminization of the Monarchy 1780-1910. Royal Masculinity and Female Empowerment, in: Andrzej Olechnowicz (Hg.), The Monarchy and the British Nation 1780 to the Present, Cambridge 2007, S. 76-107, hier S. 84.

23 Zur Amerikanischen Revolution siehe die in Anm. 5 aufgeführte Literatur. Für das Folgende speziell auch: Volker Depkat, Die Erfindung der republikanischen Präsidentschaft im Zeichen des Geschichtsbruchs. George Washington und die Ausformung eines demokratischen Herrscherbildes, in: Zeitschrift für Geschichtswissenschaft 56 (2008), S. 728-742. 
dem Rahmen der britischen Verfassung ausbrachen und ihren Protest auf eine ganz neue Grundlage stellten: Sie beriefen sich fortan auf einen naturrechtlich begründeten Liberalismus, der ganz in individuellen Grundrechten ankerte, die zu schützen die einzig legitime Verwendung staatlicher Macht war. Damit einher ging der Wandel vom Partikularismus hin zu einem Universalismus: Es waren nicht länger die ,Rechte der Engländer', auf die sich die nordamerikanischen Kolonisten im Streit mit dem Mutterland beriefen, sondern die immer und überall gültigen Menschenrechte.

Das zentrale Dokument der revolutionären Wende ist die Declaration of Independence vom 4. Juli 1776, die in sich selbst ein revolutionäres Dokument ist. ${ }^{24}$ In ihr sind die politisch-sozialen Grundprinzipien niedergelegt, auf die die künftige staatliche Ordnung der Vereinigten Staaten gebaut sein sollte. Die Unabhängigkeitserklärung proklamierte die naturrechtlich begründete Freiheit eines jeden einzelnen Individuums und sah den Schutz der individuellen Grundrechte als einzigen legitimen Staatszweck an. Sie verfügte ferner, dass die Regierungen von der Zustimmung der Regierten abhängig sein sollten und erklärte es zur gesellschaftlichen Pflicht, Herrschaftsordnungen, die den Staatszweck des Grundrechteschutzes nicht länger erfüllten, durch eine andere Ordnung zu ersetzen, die die Grundrechte besser schützen würde, als die bestehende. Damit postulierte die Unabhängigkeitserklärung nicht nur Individualismus, Konstitutionalismus und Volkssouveränität, sondern auch das Recht auf Revolution.

Der revolutionäre Gründungsakt der USA ging einher mit einer anti-monarchischen Wende. Bis zum 4. Juli 1776 hatte sich der Streit zwischen dem Mutterland und den Kolonien als ein Konflikt zwischen Parlamenten entfaltet. In ihm hatten die Kolonisten ihre ganzen Hoffnungen auf den britischen König gesetzt, von dem sie erwarteten, dass er als Sachwalter der angestammten britischen Verfassung eine Art Schiedsrichterrolle spielen würde, um das in ihren Augen außer Rand und Band geratene Parlament in London wieder zur Räson zu bringen. Genau das tat Georg III. jedoch nicht. Er tat vielmehr alles, um die vom Parlament beschlossenen Steuergesetze durchzusetzen und den Widerstand der Kolonisten zu brechen. Nicht zuletzt deshalb wälzten die Amerikaner in ihrer Unabhängigkeitserklärung, die sich in ihrem dritten Teil wie eine Anklageschrift gegen Georg III. liest, die ganze Schuld auf die britische Monarchie ab. Seitdem war die Monarchie in den Augen der Amerikaner eine von Natur aus despotische Herrschaftsform, die Freiheit systematisch zu vernichten trachtete. Mit dem 4. Juli 1776 war nicht nur die Person König Georgs III., sondern die Institution der Monarchie als solche vollständig diskreditiert.

24 Zur Unabhängigkeitserklärung hier nur: Pauline Maier, American Scripture. Making the Declaration of Independence, New York 1997; Israel, 2010 (wie Anm. 6), S. 452-460. 
Das lässt sich in der Entwicklung der revolutionären Festkultur mit Händen greifen. ${ }^{25}$ Bis in die 1770er Jahre hinein hatten die Bewohner der Kolonien in Britisch-Nordamerika in ihren diversen Fast- und Danktagen, Toasts und Ansprachen, Straßentheateraufführungen und Prozessionen zu den Freiheitsbäumen den britischen König als Wächter der britischen Verfassung gefeiert und angerufen. Nach der Unabhängigkeitserklärung etablierte sich rasch ein neuer Festkalender, der von Ritualen der symbolischen Tötung des Monarchen strukturiert war. Nach der Unabhängigkeitserklärung rissen New Yorker Bürger das Reiterstandbild Georgs III. zu Boden und hackten es in Stücke. Anderswo wurden Bilder des Monarchen verbrannt und das traditionelle Königsgeburtstagsfest wurde in die Beerdigung des Königs umfunktioniert.

Was bedeutete diese anti-monarchische Wende der Amerikanischen Revolution, die nicht nur die Monarchie, sondern überhaupt jede Form der Exekutive diskreditiert hatte, für die Ikonographie der Macht in der amerikanischen Demokratie als erste postrevolutionäre Ordnung der Moderne?

Folgen wir dem amerikanischen Schriftsteller Washington Irving, nicht viel. In seiner berühmten Kurzgeschichte „Rip van Winkle“ von 1819 erzählt er die Geschichte eines gleichnamigen Bauern, der zur Zeiten der britischen Kolonialherrschaft in einer niederländischen Siedlung im Hinterland New Yorks lebt. ${ }^{26}$ Eines Tages geht er in den Wald und fällt dort in einen tiefen, zwanzig Jahre währenden Zauberschlaf. Als er wieder aufwacht, ist die Amerikanische Revolution vorüber und Rip van Winkle - ein typisches Märchenmotiv - aus der Zeit gefallen. Er kehrt in sein Dorf zurück und findet alles grundlegend verändert vor. Viele neue Häuser sind in der Zwischenzeit gebaut worden, sein eigenes Haus liegt verfallen da und er sieht kein bekanntes Gesicht mehr. Überall trifft er auf die Zeichen einer neuen demokratisch-egalitären Zeit. Als er zu seinem ehemaligen Lieblingsort der Dorfkneipe kommt, stellt er fest, dass hier eine neues Union Hotel entstanden ist. Der ehemalige große Baum davor ist einem Fahnenmast gewichen, an dem die Stars and Stripes im Wind wehen. All' dieser Wandel ist für Rip Van Winkle schwer zu verstehen, doch dann entdeckt er sehr zu seiner Freude, das ihm zunächst vertraut erscheinende Porträt König Georgs III. Doch auch das ist seit der Amerikanischen Revolution nicht mehr dasselbe. Irving beschreibt das so:

He recognized on the sign, however, the ruby face of King George, under which he had smoked so many a peaceful pipe; but even this was singularly metamorphosed. The red coat was changed for one of blue and buff, a sword was held in the hand

25 David Waldstreicher, In the Midst of Perpetual Fetes: The Making of American Nationalism 1776-1820, Chapel Hill, N.C. 1997, S. 17-52.

26 Washington Irving: Rip van Winkle [1819]. http://www.ibiblio.org/ebooks/Irving/Winkle/Irving_Winkle.pdf (Letzte Einsicht: 30.03.2014). 
instead of a scepter, the head was decorated with a cocked hat, and underneath was painted in large characters, General Washington. ${ }^{27}$

Folgen wir dieser Geschichte, so wurde bei der visuellen Erfindung der amerikanischen Präsidentschaft also nur der rote Mantel des britischen Königs durch einen blau-beigen ausgetauscht, das Zepter durch ein Schwert und die Krone durch ein Dreispitz ersetzt. Der Rest des königlichen Porträts wurde demnach recycelt und mit einer neuen Bildunterschrift versehen.

Ganz so einfach war es freilich nicht, aber womit Washington Irving Recht hat, ist, dass sich die postrevolutionäre Ikonographie exekutiver Macht in der frühen amerikanischen Republik nicht unter dem kompletten Bruch mit der monarchisch-aristokratische Tradition formierte. Vielmehr stellt sich der Prozess der, visuellen Erfindung der amerikanischen Demokratie als ein hoch komplexer Prozess von produktiver Anverwandlung und behutsamer Demokratisierung der monarchisch-aristokratischen Tradition dar. ${ }^{28}$ Diese These möchte ich nun am Beispiel dreier ausgewählter visueller Repräsentationen des ersten amerikanischen Präsidenten diskutieren, wobei es zunächst um die Darstellung George Washingtons als Inhaber des amerikanischen Präsidentenamtes gehen soll und dann um Darstellungen von Washington als General der Kontinentalarmee, die ebenfalls im Bildhaushalt der frühen amerikanischen Republik omnipräsent waren.

\section{Visualisierung Postrevolutionärer Legitimität}

Das Urbild des postrevolutionären Staats- und Regierungschefs im Zeitalter der Demokratie stammt von Gilbert Stuart. Es ist das sogenannte „Landsdowne Portrait“ von George Washington aus dem Jahr 1796. ${ }^{29}$ Dieses Bild, von dem Stuart mehrere Kopien anfertigte, war im April 1796 von Senator William Bingham (Pennsylvania) in Auftrag gegeben und dem britischen Premierminister William Petty Fitz Maurice, $2^{\text {nd }}$ Earl of Shelburne, $1^{\text {st }}$ Marquess of Lansdowne, als Geschenk überreicht worden. In

27 Irving (wie Anm. 26), S. 19 [Hervorhebung im Original].

28 Vgl. für das folgende auch: Volker Depkat, The Grammar of Postrevolutionary Visual Politics. Comparing Presidential Stances of George Washington and Friedrich Ebert, in: Udo J. Hebel / Christoph Wagner (Hg.), Pictorial Cultures and Political Iconographies. Approaches, Perspectives, Case Studies from Europe and America, Berlin 2011, S. 176-197

29 Das Folgende im Kern nach: Volker Depkat, Freiheitsstreben und Ordnungsverlangen. Die Paradoxien der Aufklärung im Lichte visueller Narrative, in: Luise Güth u. a. (Hg.), Wo bleibt die Aufklärung? Aufklärerische Diskurse in der Postmoderne, Stuttgart 2013, S. 17-35, hier S. 28-31; Zu dem „Landsdowne Portrait" Gilbert Stuarts vgl. Carrie Rebora Barratt / Ellen G. Miles, Gilbert Stuart, New York 2004, S. 166-183; Dorinda Evans, The Genius of Gilbert Stuart, Princeton 1999, S. 60-73; Noble E. Cunningham, Popular Images of the Presidency. From Washington to Lincoln, Columbia, MO 1991, S. 130-139. 
Form von Stichen und Drucken war das Bild vielfältig in den USA - aber auch in Europa - präsent. Das zeigt nicht zuletzt der hier abgedruckte Stich „General Washington", der im Jahre 1818 von C. Goodman und B. Piggot in Philadelphia zum Verkauf auf dem damals rasch expandierenden Markt für Stiche angefertigt wurde (Abb. 3).

Washington steht im Zentrum des monumentalen Bildes. Sein Körper ist im halblinken Profil ganz zu sehen, sein rechter Arm ist in Rednergeste ausgestreckt, in seiner linken Hand hält er ein Zierschwert in der Scheide, dessen Spitze auf den Boden zeigt. Das Schwert deutet auf seine in der Vergangenheit liegenden militärischen Verdienste hin, doch bleibt der, General' George Washington in diesem Bild ganz klar der Zivilperson des ,Präsidenten' George Washington untergeordnet. Washington hat einen dunklen Samtanzug an, dessen Jacke mit Stehkragen bis auf seine Knie herab reicht. Dazu trägt er schwarze Strümpfe, schwarze Lederschuhe mit silbernen Schnallen und ein weißes Hemd mit Spitzenborten an Kragen und Ärmeln. Insgesamt ist seine Kleidung von unaufdringlicher Eleganz. Sie ist zwar von erkennbar guter Qualität, aber nicht pompös oder extravagant, sondern eher moderat, zurückgenommen und bürgerlich. Ein schwarzer Hut mit Kokarde, der auf dem Schreibtisch liegt, komplettiert seinen formal korrekten Anzug. Der Hut des Bürgers ist an die Stelle der monarchischen Krone getreten.

Das Gesicht Washingtons ist symmetrisch geformt und ebenmäßig proportioniert. Er wirkt wie eine antike Statue und verrät Milde und Entschlusskraft zugleich. Vor dem Hintergrund physiognomischer Theorie wird sein ,schönes' Gesicht zum Spiegel seiner ,schönen' Seele - und wir wissen, wie sehr Gilbert Stuart sich angestrengt hat, dem in Wirklichkeit sehr viel grobschlächtigeren Gesicht Washingtons die engelsgleiche Ebenmäßigkeit zu geben. ${ }^{30}$ Politisch gewendet heißt das, dass das Gesicht Washingtons den tugendhaften und moralischen Charakter des ersten amerikanischen Präsidenten visualisiert, ohne den im Denken der Zeit ein auf Freiheit und Selbstbestimmung gegründetes Gemeinwesen nicht bestehen konnte. ${ }^{31}$

Betont sei, dass Washington hier als Bürger des Staates dargestellt wird, dem er als Oberhaupt und Regierungschef vorsteht. Der fiktive Raum, in dem er sich befindet, ist offen und transparent. Anders als Georg II. steht George Washington nicht erhöht auf einem Podest, sondern bleibt buchstäblich auf dem Teppich. Seine ausgestreckte Hand scheint anzudeuten, dass er als Redner versucht, sein Publikum zu überzeugen und somit mit der Kraft des besseren Argumentes zu regieren. Dabei ordnet er sich stets der Verfassung als Quelle seiner temporären Macht unter. Das kommunizieren vor allem die Bücher, die unter und auf dem Schreibtisch durcheinander liegen. Eines der Bücher unter dem Tisch heißt „Constitution \& Laws of the United States“, und auf dem Schreibtisch liegen „The Federalist Papers“ und „The

30 Barrat / Miles, 2004 (wie Anm. 29), S. 147-153.

31 Depkat, 2008 (wie Anm. 23), S. $738 f$. 
Journal of Congress“..$^{32}$ All diese Werke sehen benutzt aus; der Präsident arbeitet also mit ihnen. Die Schriftstücke auf dem Schreibtisch und das Tintenfass mit Feder weisen Washington als einen persönlich regierenden Staatsmann aus, der durch seine Tätigkeit die Verfassungsordnung erhält und in die Zukunft sichert.

Individuelle Leistung als Ausweis moralischer Integrität wird somit zum zentralen Kriterium von Washingtons Autorität und Legitimität. Das wird auf dem Bild auf vielfache Weise visualisiert. Zwei der Bücher unter dem Tisch - ein Werk mit dem Titel „American Revolution“ und die von ihm als Oberkommandierenden der Kontinentalarmee erlassenen Tagesbefehle (General Orders) - verweisen direkt auf Washingtons Verdienste um die Amerikanische Revolution und lassen ihn gleichsam zur Personifikation der in der Unabhängigkeitserklärung und der Verfassung von 1787 eingeschriebenen, politisch-sozialen Wertideen erscheinen. Ebenso verweist das Zierschwert auf seine militärischen Erfolge im Amerikanischen Revolutionskrieg. Allerdings entwirft die klare Unterordnung der militärischen unter die zivile Symbolik Washington als Cincinnatusfigur, als einen idealen Republikaner also, der in Zeiten der äußeren Bedrohung seine privaten Interessen hintanstellt und das bedrohte Gemeinwesen unter Einsatz seines Lebens verteidigt, nur um wieder ins Privatleben zurückzukehren, sobald die Gefahr abgewendet ist. ${ }^{33}$

Wenngleich die Darstellung Washingtons als erstes postrevolutionäres Staatsoberhaupt und Regierungschef im „Landsdowne Portrait“ noch unverkennbar viele Elemente der monarchisch-aristokratischen Ikonographietraditionen aufweist, so ist es doch bemerkenswert, dass Gilbert Stuart sich entschloss, Washington ostentativ als ersten Bürger des Staates zu entwerfen, der auch im Amt des Präsidenten stets Teil der Gesellschaft bleibt, die er regiert. Dominant sind Symbole von Staatsbürgerlichkeit und bürgerlicher Respektabilität, die auf persönlicher Bildung, individueller Leistung, Tugend und Moral beruht.

Ungeachtet der prägenden Wirkung, die Stuarts Darstellung des Zivilisten George Washington auf dem „Landsdowne Portrait“ für die visuelle Kultur der amerikanischen Präsidentschaft hatte, gab es in der frühen amerikanischen Republik ungleich mehr visuelle Repräsentationen, die Washington als General und siegreichen Feldherrn darstellten. ${ }^{34}$ Washington war nun einmal erfolgreicher General bevor er Präsident wurde - und er wurde wohl auch nur Präsident, weil er von der überwiegenden Mehrheit der Zeitgenossen wegen seiner in scheinbar selbstlosem Einsatz für die Sache erbrachten militärischen Verdienste als Personifizierung der Amerikanischen

32 Barrat / Miles, 2004 (wie Anm. 29), S. 169.

33 Vgl. dazu Garry Wills, Cincinnatus. George Washington and the Enlightenment, New York 1984; Karsten Fitz, The American Revolution Remembered, 1830s-1850s. Competing Images and Conflicting Narratives, Heidelberg 2010.

34 Mark E. Thistlethwaite, The Image of George Washington. Studies in Mid-Nineteenth-Century American History Painting, New York 1979. 


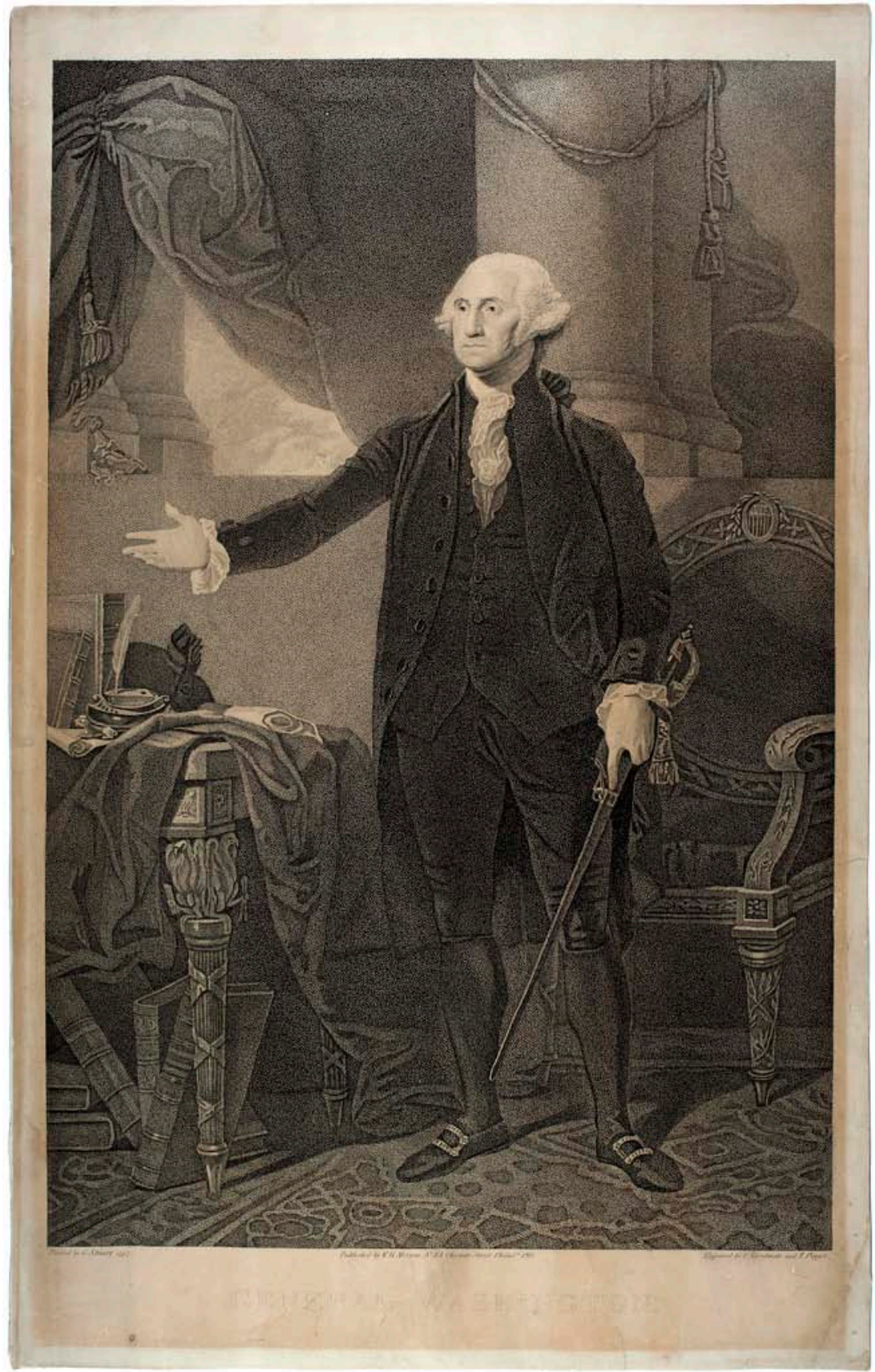

Abb. 3: Gilbert Stuart (Maler)/C. Goodman und B. Piggot (Stecher/Drucker), General Washington, Philadelphia 1818.

American Antiquarian Society, R54 B dr 6 - CAPE. 


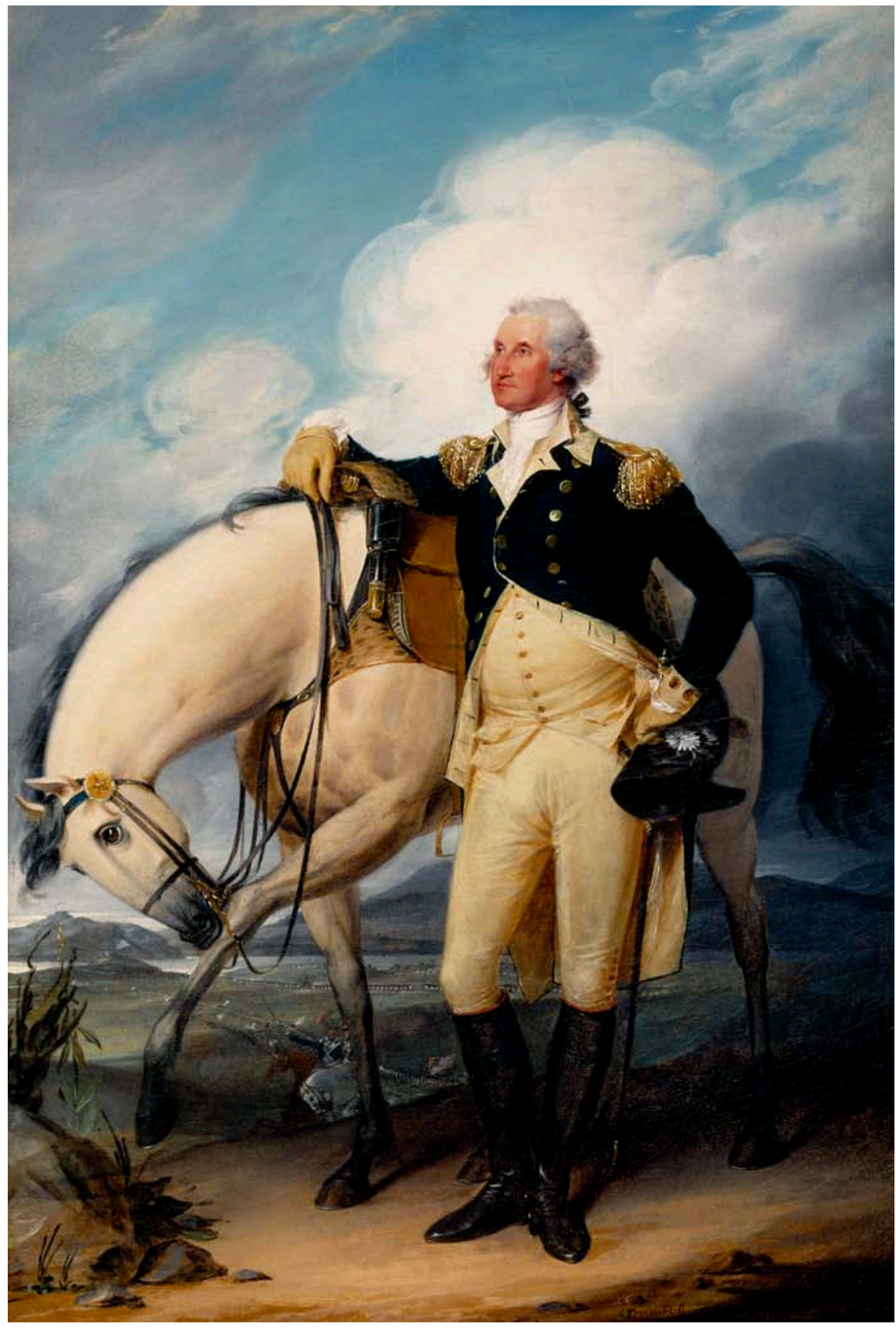

Abb. 4: John Trumbull, Washington at Verplanck's Point, 1790.

Winterthur Museum, 1964.2201. 
Revolution gesehen wurde. ${ }^{35}$ Ein auf militärisches Charisma gestützter Führungsanspruch ist für die ,politische Kultur' der amerikanischen Präsidentschaft bis weit ins 20. Jahrhundert hinein prägend gewesen. Gleichzeitig setzt sich hier eine im Zusammenhang mit Georg II. diskutierte monarchisch-aristokratische Tradition fort, die allerdings im Prozess der Ausgestaltung der amerikanischen Republik demokratisiert wurde. Auch dies hat seinen Niederschlag in der visuellen Kultur der amerikanischen Präsidentschaft gefunden.

Nehmen wir zum Beispiel das Gemälde „Washington at Verplanck's Point“ von John Trumbull aus dem Jahre 1790 (Abb. 4). ${ }^{36}$ Der führende Washington-Porträtist seiner Zeit hat dieses Bild zur Freude und Erbauung von Martha Washington gemalt, die dem Maler in der Vergangenheit zu mehreren Sitzungsterminen mit ihrem Ehemann George verholfen hatte. Auch für dieses Gemälde hatte Washington persönlich Modell gesessen. „Washington at Verplanck's Point“ hing zunächst im New Room von Washingtons Wohnsitz Mount Vernon und ging dann nach dem Tod von Martha Washington in den Besitz der Enkelin Elizabeth Custis Law über. ${ }^{37}$ Gleichwohl zirkulierte dieses Motiv breit in der frühen Republik, weil einerseits Trumbull selbst mehrere Kopien von diesem Bild in Umlauf brachte und weil sich andererseits in der ersten Hälfte des 19. Jahrhunderts ein rasch expandierender Markt für Lithographien etablierte, auf dem sich mit Drucken von George Washington gute Geschäfte machen ließen.

Das Bild zeigt eine Szene aus dem Jahr 1782. General Washington steht in Uniform ohne Kopfbedeckung neben seinem Schimmel. Sein rechter Arm ist abgewinkelt auf den Sattel gelehnt, in der Hand hält er locker die Zügel des sich bewegenden Pferdes, das sich mit dem Maul das linke Bein kratzt oder leckt. Washington ist anmutig und wirkt entspannt, hat aber doch die volle Kontrolle über das Pferd, das merkwürdig klein erscheint. Reiter wissen, dass eine solche, auf das Pferd gestützte lockere Pose ein Ding der Unmöglichkeit ist, was nur zu unterstreichen scheint, dass es Trumbull hier primär um die Visualisierung von Führungskraft und Herrschaft geht.

Washington steht auf einer Anhöhe über dem Tal des Hudson River, von wo er die im Hintergrund des Bildes ablaufenden Vorgänge zu beobachten scheint. Dort

35 Mark E. Thistlethwaite, The Face of the Nation. George Washington's Image and American Identity, in: Depkat / Zwingenberger, 2012 (wie Anm. 11), S. 35-52; Glenn A. Phelps, George Washington and American Constitutionalism, Lawrence, KS 1993, S. 102.

36 Zu Trumbull, dem Maler der Amerikanischen Revolution: Irma B. Jaffe, John Trumbull. Patriot-Artist of the American Revolution, Boston 1975; Dies., Trumbull. The Declaration of Independence, New York 1976; Helen A. Cooper, John Trumbull. The Hand and Spirit of a Painter, New Haven, CT 1982; Stuart A. P. Murray, John Trumbull. Painter of the Revolutionary War, Armonk, NY 2009; J. F. Weir, John Trumbull. A Brief Sketch of His Life, to which is added a Catalogue of his Works, New York 1901.

37 Vgl. Amanda Isaac, Furnishing the New Room: John Trumbull's Portrait of Washington. http://mountvernonnewroom.tumblr.com/post/52946632321/furnishing-the-new-room-john-trumbulls-portrait-of (Letzte Einsicht: 30.03.2014); Cooper, 1982 (wie Anm. 36), S. $118 f$. 
sieht man das Feldlager der amerikanischen Kontinentalarmee am Verplanck's Point, ebenso die von der Schlacht von Yorktown zurückkehrenden französischen Einheiten, die über die Hügel des Flusstales in das Lager der Amerikaner einmarschieren, wo sie freudig begrüßt werden. Wir sehen Washington also als Schlachtensieger nicht lange nach der britischen Kapitulation in Yorktown am 19. Oktober 1781.

Vergleicht man diese Darstellung des siegreichen Generals mit dem oben erörterten Reiterstandbild von Georg II. werden einige Grundmuster der in den USA neu entstehenden Ikonographie der Macht sichtbar. Washington steht neben dem Pferd und sitzt nicht auf ihm. Zwar kontrolliert er das Pferd mit relativer Lässigkeit, doch lässt er ihm zugleich seine Bewegungsfreiheit. Insgesamt erscheint Washington hier nicht als Triumphator, sondern nimmt sich und seine Person sehr zurück. Er wirkt wie ein stiller Genießer der Szene unter ihm. Die Uniform ist eher schlicht, hat nur wenig Dekor, keine Ehrenzeichen oder sonstige Insignien. Insgesamt entwirft Trumbull Washington hier als einen Bürgergeneral, der nun, nachdem das militärische Werk vollendet ist, bald wieder ins Privatleben zurückkehren wird. Washington ist hier ganz gewiss kein Ritter in antikisierend-feudaler Tradition wie Georg II., sondern eher ein römischer Civis, der sich in Zeiten der äußeren Bedrohung selbstlos in den Dienst der Republik gestellt hat, um die Freiheit zu verteidigen. Damit lässt sich das Gemälde „Washington at Verplanck’s Point“ als eine andere Visualisierung des Cincinnatus-Themas lesen, das für die Ausgestaltung der amerikanischen Demokratie allgemein und insbesondere der amerikanischen Präsidentschaft von überragender Bedeutung ist.

Werfen wir deshalb noch einen abschließenden Blick auf eine dreidimensionale visuelle Repräsentation George Washingtons, nämlich die Statue des französischen Bildhauers Jean-Antoine Houdon, die seit 1796 in der Rotunda des State Capitolvon Virginia steht (Abb. 5). ${ }^{38}$ Sie wurde 1784 vom Parlament des Bundesstaates bei Houdon in Auftrag gegeben. Houdon begann mit den Arbeiten im Jahr 1785 und war 1791/92 mit der Skulptur fertig. 1796 lieferte er sie aus. Der französische Bildhauer hatte Washington persönlich getroffen, eine Gesichtsmaske von ihm abgenommen und auch den Körper des Revolutionshelden vermessen. Deshalb gilt diese Statue als eine der akkuratesten Darstellungen George Washingtons.

Die aus Carrara Marmor gefertigte Skulptur zeigt einen stehenden Washington in Lebensgröße. Washington trägt seine Uniform, doch hält er in der rechten Hand einen Spazierstock, während sein linker Arm auf einem Fascis ruht, einem Rutenbündel, das im Römischen Reich das Symbol der höchsten Amtsträger war. An dem

38 Charles H. Hart / Edward Biddle, George Washington, Jean Antoine Houdon, Sculptor. A Brief History of the Most Famous Sculpture Created of America's Immortal Patriot. Issued to Commemorate the Bicentennial of His Birth, 1732-1932, Providence, R.I., 1931; Ronald E. Heaton, The Image of Washington. The History of the Houdon Statue, Norristown, PA 1971; Allgemein zu Houdon: Anne L. Poulet, Jean-Antoine Houdon. Sculptor of the Enlightenment, Washington, DC 2003. 


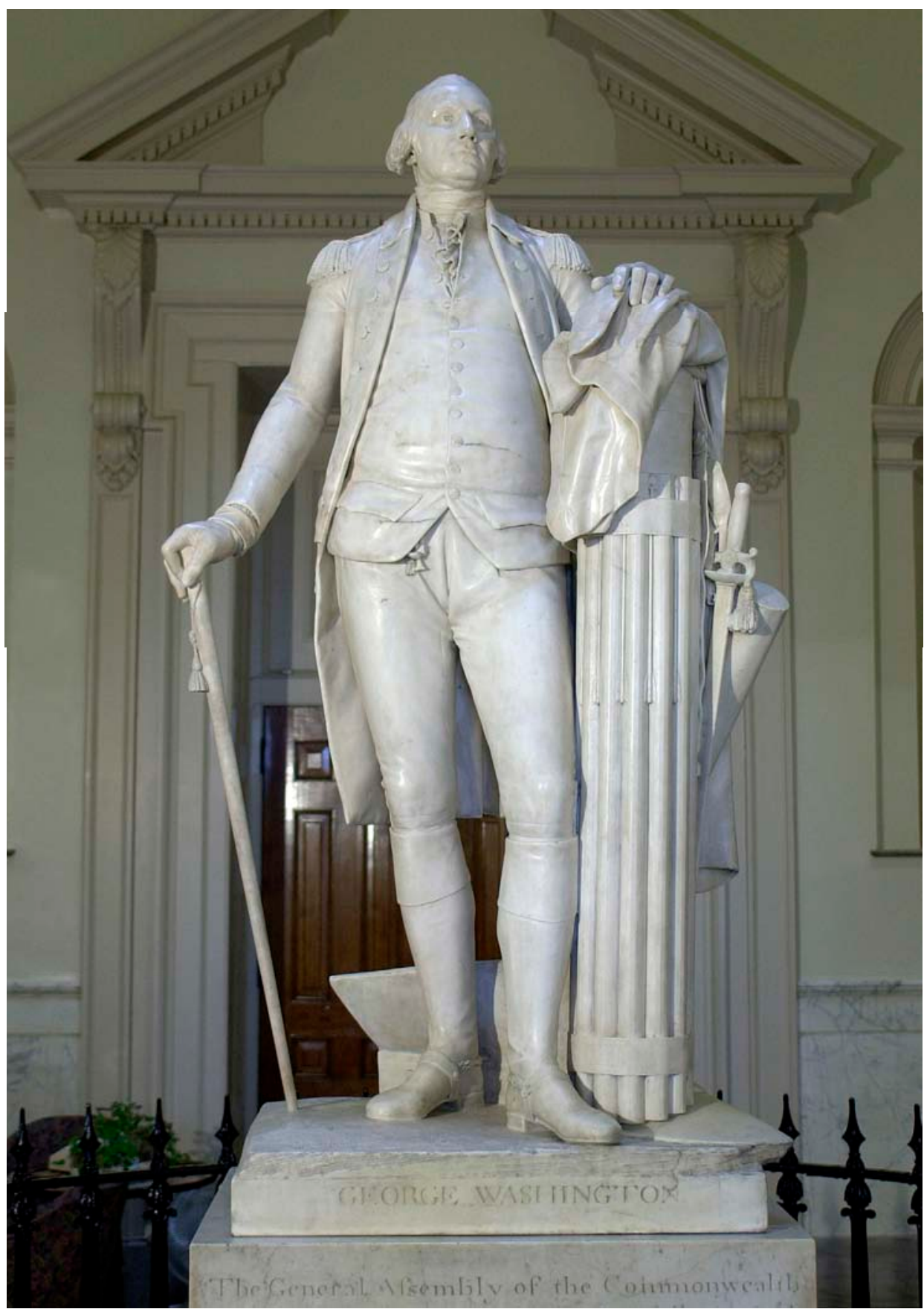

Abb. 5: Jean-Antoine Houdon, George Washington, 1785-1792.

State Artwork Collection, Library of Virginia. www.virginiamemory.com 
Rutenbündel hängen Washingtons Umhang und sein Schwert, und hinter dem Fascis ist ein Pflug zu erkennen. Damit zeigt die Statue Houdons Washington als römischen Bürgersoldaten im Moment des Übergangs von der soldatischen zur zivilen Existenz. Die Ausnahmesituation des Krieges ist vorbei; Washington hat das Schwert buchstäblich an den Nagel gehängt und mit dem Spazierstock schon wieder einen zivilen Gegenstand in der Hand, der ihn zugleich als Gentleman ausweist. Der Pflug im Hintergrund zeigt an, dass Washington eigentlich ein Farmer ist und somit auch in das Leben als Farmer zurückkehrt. Damit liefert diese Statue eine Visualisierung des abstrakten Ideals vom gentleman farmer, der als idealer Republikaner in römisch-antiker Tradition im Moment äußerer Gefahr seinen Pflug gegen das Schwert eintauscht, um die republikanische Freiheit zu verteidigen, und der nun, nachdem die Gefahr abgewendet ist, wieder in sein ziviles Leben als Landwirt zurückkehrt. Die revolutionär begründete amerikanische Demokratie machte in der Ausgestaltung ihrer politischen Kultur mithin produktive Anleihen beim klassisch-römischen Republikanismus, indem sie das Bild vom königlichen Feldherrn in das antik-römische Ideal des Cincinnatus umformte, um die Legitimität exekutiver Macht zu kommunizieren, und das nicht nur visuell. ${ }^{39}$

\section{Schluss}

Wenn die Bewohner der britischen Kolonien in Nordamerika sich vorstellten, wie politische Macht aussehe und woran man sie erkenne, dann hatten viele von ihnen bis zur Revolution mit Sicherheit Bilder der hannoverschen Kurfürsten auf dem englischen Thron vor Augen. Die offiziellen Porträts von Georg I., Georg II. und Georg III. waren in Form von kopierten Gemälden auch in den Kolonien allgegenwärtig und erreichten in Form von Drucken noch ein viel weiteres Publikum, so begrenzt das aus heutiger Sicht auch war. Als Akte visueller politischer Kommunikation vermittelten Krönungsporträts, Reiterbilder und andere visuelle Darstellungen der britischen Könige Vorstellungen von exekutiver Macht und legitimer politischer Herrschaft. Bis zum 4. Juli 1776 war die Ikonographie der Macht in den nordamerikanischen Kolonien des britischen Weltreiches von Herrschaftszeichen der Monarchie und einem aristokratisch-feudalen Symbolhaushalt geprägt.

Die Amerikanische Revolution und ihr radikaler naturrechtlicher Egalitarismus entfalteten sich als totaler Bruch mit der Monarchie als Herrschaftsform. Das am 4. Juli 1776 begonnene Experiment in Demokratie sah sich deshalb einem potentiellen symbolischen Vakuum gegenüber, wenn es darum ging, das historische No-

39 Georg Schild, Res Publica Americana. Romrezeption und Verfassungsdenken zur Zeit der Amerikanischen Revolution, in: Historische Zeitschrift 284 (2007), S. 31-58. 
vum einer flächenstaatlichen Demokratie, die als moderner Verfassungsstaat in allen ihren Teilen auf dem Prinzip der Volkssouveränität gegründet war, auch visuell zu legitimieren. Dabei waren die Befürworter der Verfassung von 1787 gezwungen, die neue Ordnung gleich an zwei Fronten visuell zu verteidigen und ihre Legitimität zu behaupten, und zwar einerseits gegenüber der europäischen aristokratisch-feudalen Tradition und andererseits gegenüber den Kritikern in den USA selbst, die die föderal-repräsentative Verfassung von 1787 als Bruch mit den Idealen der Unabhängigkeitserklärung und ihrem radikal-demokratischen Potential interpretierten. Diese doppelte Frontstellung umreißt das Spannungsfeld, in dem die visuelle Kultur der amerikanischen Präsidentschaft erfunden wurde, wobei hier eine Vielzahl von Akteuren zusammenwirkten: die Präsidenten selbst, die Maler, die Drucker und nicht zuletzt diejenigen Amerikaner, die als Käufer der Präsidentendarstellungen den durchaus lukrativen Markt für die Bilder von amerikanischen Präsidenten definierten.

Insgesamt stellt sich die visuelle Erfindung der amerikanischen Präsidentschaft nicht als abrupter Bruch mit den europäischen Traditionen dar, sondern vielmehr als ein gradueller Umbau europäischer Traditionen, die produktiv adaptiert und neu formuliert wurden. Damit lässt sich die visuelle Erfindung der amerikanischen Präsidentschaft einerseits als Anverwandlung römisch-antiker ikonographischer Traditionen, andererseits als schrittweise Demokratisierung insbesondere der monarchisch-aristokratischen Ikonographie beschreiben, die für die Generation der amerikanischen Revolutionäre unmittelbar durch Georg I., Georg II. und Georg III. konkretisiert wurde. In diesem Zusammenhang lässt sich die Konstruktion einer Ikonographie demokratischer Macht durchaus als europäisch-amerikanisches Gemeinschaftsprodukt begreifen.

Gilbert Stuart war ein in England geborener und ausgebildeter Maler, der, fasziniert von den USA, in den 1790er Jahren ganz bewusst nach Amerika segelte, um Washington als Held der Revolution und erstes postrevolutionäres Staatsoberhaupt zu malen. Jean-Antoinne Houdon, dem wir die wohl wirklichkeitsgetreueste Darstellung George Washingtons verdanken, war Franzose, der sich ebenfalls von den USA begeistert zeigte und seine eigenen Hoffnungen auf eine bessere Ordnung auf sie und insbesondere George Washington projizierte. Damit steht die Ausgestaltung einer Ikonographie demokratischer Macht am Beginn einer viel umfassenderen Entwicklung, in deren Verlauf Amerikaner und Europäer immer wieder gemeinschaftlich Bilder von Amerika entwarfen und Amerika so zusammen erfanden. 